\title{
Exposure to Underrepresentation Discussion: The Impacts on Women's Attitudes and Identities
}

\author{
Geoff Potvin*, Zahra Hazari ${ }^{\dagger}$ and Robynne M. Lock** \\ *Department of Physics, and STEM Transformation Institute, Florida International University, 11200 SW $8^{\text {th }}$ \\ St., Miami, FL 33199 \\ ${ }^{\dagger}$ Department of Teaching \& Learning, Department of Physics, and STEM Transformation Institute, Florida \\ International University, 11200 SW $8^{\text {th }}$ St., Miami, FL 33199 \\ ${ }^{* *}$ Department of Physics \& Astronomy, Texas A \& M University - Commerce, 2200 Campbell St., Commerce, TX \\ 75428
}

\begin{abstract}
In earlier work, we found that women who reported experiencing the "discussion of underrepresentation" in their high school physics classes were more likely to report a career interest in the physical sciences in college. To explore this effect in more detail, we conducted two double-blind, random-assignment experiments on students enrolled in introductory, algebra-based college physics. In the first, students were randomly assigned to read one of two short essays (one focused on the underrepresentation of women in physics, the other reporting on AMO physics research led by a woman) followed by a uniform set of reflection questions. In the second, another cohort of students was primed on their beliefs about gender differences in physics before undergoing the same treatment (one of two essays and reflection questions). In this paper, we compare the impacts on students' general science identity, physics identity, and performance gains on selected FMCE problems.
\end{abstract}

Keywords: Gender issues, physics identity

PACS: 01.30.Cc, 01.40.Fk

\section{MOTIVATIONS}

Identity has proven to be a relevant framework for understanding student attitudes towards physics $[1,2]$ and may help to explain why the participation of women in physics has remained remarkably low, with bachelor's degree recipients stagnating around $22 \%$ representation of women. Identity is conceptualized as being influenced by students' interest and recognition beliefs (with an indirect, mediated relationship to their performance/competence beliefs as well) [2]. It has been shown to be predictive of students' physical science career interest, with women on average holding much weaker physics identities than men. It has also proven to be a useful framework for understanding students' career choices in other domains including mathematics [3] and engineering [4].

In other work, we previously tested five common hypotheses on how to engage women in physics more effectively including single sex classrooms, having a female teacher or other female science role models, talking about the history of women in science, and talking about underrepresentation [5]. Using very robust matching methods on cross-sectional data to control for differences on several confounding covariates, we found that only the latter factor had a significant impact on women's physical science career intentions; the first four had no significant relationship with women's intentions. The im- pacts of this type of experience had been noted elsewhere in large-scale studies [1] as well as in classroom observational work [6], but the specific mechanisms by which this happens and the size of the impact (relative to the level of exposure received by students) have not been understood. In this paper, we report on two, related studies that probed these considerations.

\section{STUDY 1}

In order to explore the effects of exposure to discourse on underrepresentation further and to possibly develop a practical exercise that could be used within the constraints of a large classroom or with short time constraints, in the fall semester of 2012 we conducted a double-blind experiment. We set out to determine the effect of such exposure on students' physics identities, as well as their general science identities and performance on a subset of questions from the Force and Motion Conceptual Evaluation (FMCE) [7]. Student participants were recruited from a large (200+) section of introductory algebra-based physics at a southeastern public, land-grant institution taught by a male faculty member. This course was offered primarily to STEM majors (other than physical science and engineering majors) as well as to non-STEM majors as a science elective. The course content was a largely traditional survey of intro- 
ductory mechanics including Newton's laws, energy, momentum, and gravity.

The experiment was implemented inside students' online homework system. Students were offered a few points of extra homework credit for completing the assignment, and it was available during the 13th week of the 15 week semester. Appearing within the usual homework interface, students were randomly assigned (by the homework system itself) to one of two assignments in a pool. The "control" condition presented students with a short press release-type essay on cold-temperature atomic physics (prominently featuring a female physicist principal investigator while not explicitly mentioning gender anywhere), while the "treatment" condition presented a similar-length essay on underrepresentation in physics. Both essays were approximately 200 words in length and had been edited for brevity from the original, publicly available sources. In both conditions, after reading the essay, students were prompted to answer three open-ended questions:

1. What is the point of this essay?

2. What does this essay tell you about physics?

3. In what ways, if any, does reading this essay change how you think about physics?

These were intended to prompt students to reflect on the readings without directly addressing the content (and to provide uniformity across the conditions). We hypothesized that students, especially women, might undergo an improvement in their physics identities after having been exposed to a short discourse on representation problems in the physics community and/or show a corresponding gain in their physics performance (through a reduction in physics-related anxiety in the spirit of stereotype threat neutralization $[8,9]$ ).

As outcomes, we assessed students' physics identity using our previously-developed physics identity instrument, which also includes items that assess students' general science identities in a similar format [10]. Items on this instrument take the form of anchored, 7-point scales and include 15 physics identity items and 13 general science identity items, which are combined together (and normalized to be scored out of a total of 100) to form two different proxies for these two identities. Further, we also assessed students' conceptual understanding of physics using a select subset of 11 FMCE questions [7]. Specifically, we chose questions \# 11, $12,13,22,23,24,25,26,39,44$, and 46 in order to cover a variety of introductory physics concepts in a time-constrained assessment. These two instruments were given to students at the beginning of the course and then again within a week after they had participated in the experiment. Individuals' responses were matched so that direct pre-post comparisons could be made. We also collected students' gender data to include in the analysis. In total, 96 students participated in all parts of the experiment (pre-assessment, treatment, and post-assessment), including 65 women and 31 men (consistent with the overal gender breakdown of this course). In terms of the two conditions, 40 experienced the control (29 women, 11 men) and 56 experienced the treatment (36 women, 20 men).

For our analysis ${ }^{1}$, our primary goal was to test whether students in the two conditions showed differences in their pre-post gains in physics identity, general science identity, or FMCE performance, and whether there were any gender-related effects (e.g. women may show gains while men will not, etc). As a secondary goal, we considered how students' (and, specifically, women's) identities changed over the course of most of a semester.

A descriptive summary is reported in Table 1. In terms of the overall changes from pre to post (not disaggregating by experimental condition), note that there is no significant change in terms of physics identity gain/loss overall $(t=0.78, d f=95, p>0.05)$, nor is there a significant change for women $(t=1.15, d f=65, p>$ $0.05)$ or men $(t=0.39, d f=30, p>0.05)$. Similarly, there is no significant change in science identity overall $(t=0.74, d f=95, p>0.05)$, nor for women $(t=$ $0.45, d f=64, p>0.05)$ or men $(t=0.71, d f=30$, $p>0.05)$. FMCE scores improved significantly overall $(t=4.45, d f=95, p<0.001)$ as well as for women alone $(t=3.67, d f=64, p<0.001)$ and men alone $(t=2.49, d f=30, p<0.05)$.

Disaggregating by the two conditions of the study (see Table 2), we found that the experimental condition did not significantly impact students' identities or FMCE performance. Specifically, there was no significant difference between treatment and control overall $(t=0.94, d f=75.5, p>0.05)$ nor was there a difference amongst the female participants $(t=0.19, d f=58.6$, $p>0.05)$ nor the male participants $(t=1.26, d f=14.0$, $p>0.05)$. For science identity, the two conditions were similar overall $(t=0.97, d f=93.3, p>0.05)$ as well as for women $(t=0.90, d f=62.7, p>0.05)$ and men $(t=0.39, d f=23.8, p>0.05)$ in the study. Lastly, gains on the FMCE questions were similarly non-significant overall $(t=0.86, d f=85.4, p>0.05)$ and for womenonly ( $t=0.26, d f=55.9, p>0.05$ ), although for menonly there was a marginal change $(t=2.24, d f=28.9$, $p<0.05$ ), with men in the treatment condition outperforming men in the control condition. Note, however, that these were the two smallest subgroups in the study ( $n=20$ and 11, respectively), so this may be spurious.

\footnotetext{
1 Note that all data processing and analyses have been conducted using $\mathrm{R}$ [11], and $\alpha$, the maximum acceptable likelihood of Type I error has been set at $5 \%$ throughout.
} 
TABLE 1. Descriptive summary of Study 1 participants.

\begin{tabular}{lccc}
\hline Quantity & Mean \pm SD & Women Only & Men Only \\
\hline Physics identity - PRE (/100) & $42 \pm 17$ & $38 \pm 16$ & $50 \pm 16$ \\
Physics identity - POST $(/ 100)$ & $41 \pm 19$ & $36 \pm 19$ & $51 \pm 17$ \\
\hline Science identity - PRE $(/ 100)$ & $63 \pm 16$ & $63 \pm 17$ & $62 \pm 15$ \\
Science identity - POST $(/ 100)$ & $62 \pm 16$ & $62 \pm 17$ & $61 \pm 15$ \\
\hline FMCE - PRE (/100) & $13 \pm 15$ & $12 \pm 13$ & $15 \pm 18$ \\
FMCE - POST $(/ 100)$ & $21 \pm 16$ & $19 \pm 14$ & $23 \pm 19$ \\
\hline
\end{tabular}

TABLE 2. Changes in identity and FMCE in Study 1 (Post - Pre score), disaggregated by experimental condition.

\begin{tabular}{l|ccc|ccc}
\hline \multicolumn{1}{c|}{ Quantity } & \multicolumn{3}{c|}{ Gain (Treatment) } & \multicolumn{3}{c}{ Gain (Control) } \\
& All Treatment & Women Only & Men Only & All Control & Women Only & Men Only \\
Physics identity (/100) & $0.04 \pm 11$ & $-1.5 \pm 12$ & $2.8 \pm 8.2$ & $-2.3 \pm 13$ & $-2.1 \pm 13$ & $-2.9 \pm 14$ \\
Science identity (/100) & $0.07 \pm 14$ & $0.57 \pm 15$ & $-0.83 \pm 11$ & $-2.4 \pm 11$ & $-2.4 \pm 12$ & $-2.3 \pm 9.6$ \\
FMCE (/100) & $9.3 \pm 18$ & $7.3 \pm 16$ & $13 \pm 20$ & $6.1 \pm 17$ & $8.5 \pm 19$ & $0.00 \pm 11$ \\
\hline
\end{tabular}

The across-the-board results that the treatment condition had no significant impact on students' identities or FMCE performance led us to modify the experiment in order to increase its impact. Again, as mentioned earlier, we have seen that the discussion of underrepresentation has a significant impact on women in different contexts $[5,1]$. One important difference between this experiment and other contexts may be the limited exposure to this discourse in the experiment (a few minutes reading and reflection) as well as not having any real social interaction in the process, as students were participating through their online homework system only. The gender discourse in the treatment condition may have been too disconnected from students' lives and experiences to be meaningful. For these reasons, we conducted a second experiment which attempted to bring gender issues more to the forefront of students' thoughts, while preserving the experimental design.

\section{STUDY 2}

In the spring semester of 2013, we conducted a second double-blind experiment that modified the first study in one particular way: during both the treatment and control conditions, students were first asked to answer the question "Do you think there are gender issues in physics?" before reading their randomly-assigned essay and then answering the same three reflection questions as before. The course from which students were recruited was the same introductory algebra-based physics course listing as in the first study. Also to note is that the course was taught by a different, female faculty.

A descriptive summary of the Study 2 participants is reported in Table 3. In total, there were 106 individuals who participated in the experiment (pre-assessment, treatment, and post-assessment) including 76 women and
30 men, again consistent with the gender distribution in the entire course. 46 students experienced the control condition ( 34 women, 12 men) and 60 experienced the treatment condition (42 women, 18 men). In this semester there was a marginally significant change in students' physics identity $(t=2.1, d f=105, p<0.05)$ including for women $(t=2.03, d f=75, p<0.05)$ separately but not for men on their own $(t=0.59, d f=29$, $p>0.05)$. There was also a significant change in students' science identity $(t=3.25, d f=105, p<0.01)$ including an improvement for women $(t=2.7, d f=75$, $p<0.01)$ but again not for men $(t=1.8, d f=29$, $p>0.05)$, probably due to their smaller sample size. In this population, there was a significant (and larger) improvement in FMCE scores overall $(t=7.1, d f=105$, $p<0.001)$ as well as for women $(t=6.5, d f=75$, $p<0.001)$ and men $(t=3.5, d f=29, p<0.01)$.

Nonetheless, disaggregating by the two conditions of the study (see also Table 4), we found that the experimental condition still did not significantly impact student identities or performance. In terms of physics identity gains, there was no significant difference between the two conditions $(t=0.86, d f=91.1, p>0.05)$ nor was there a difference for women $(t=0.36, d f=63.2$, $p>0.05)$ or men $(t=1.22, d f=27.2, p>0.05)$ on their own. Similarly, science identity gains were also non-significant overall $(t=0.74, d f=93.6, p>0.05)$ or when disaggregating by women $(t=0.17, d f=71.1$, $p>0.05)$ and men $(t=1.2, d f=18.6, p>0.05)$. Finally, FMCE gains were also not different between the two conditions $(t=0.23, d f=102.3, p>0.05)$ including for women-only $(t=0.63, d f=73.9, p>0.05)$ and men-only $(t=1.0, d f=26.5, p>0.05)$.

The results of this second study indicate that even though this cohort of students actually improved their physics and science identities over the course of the semester (though with quite small gains) and improved 
TABLE 3. Descriptive summary of Study 2 participants.

\begin{tabular}{lccc}
\hline Quantity & Mean \pm SD & Women Only & Men Only \\
\hline Physics identity - PRE (/100) & $39 \pm 17$ & $37 \pm 18$ & $44 \pm 15$ \\
Physics identity - POST (/100) & $42 \pm 19$ & $41 \pm 20$ & $46 \pm 16$ \\
\hline Science identity - PRE (/100) & $58 \pm 18$ & $58 \pm 19$ & $57 \pm 17$ \\
Science identity - POST (/100) & $62 \pm 19$ & $62 \pm 19$ & $61 \pm 19$ \\
\hline FMCE - PRE (/100) & $14 \pm 16$ & $14 \pm 16$ & $15 \pm 18$ \\
FMCE - POST (/100) & $27 \pm 19$ & $26 \pm 18$ & $30 \pm 23$ \\
\hline
\end{tabular}

TABLE 4. Changes in identity and FMCE in Study 2 (Post - Pre score), disaggregated by experimental condition.

\begin{tabular}{l|ccc|ccc}
\hline \multicolumn{1}{c|}{ Quantity } & \multicolumn{3}{c|}{ Gain (Treatment) } & \multicolumn{3}{c}{ Gain (Control) } \\
& All Treatment & Women Only & Men Only & All Control & Women Only & Men Only \\
Physics identity (/100) & $4.1 \pm 14$ & $4.1 \pm 14$ & $4.0 \pm 16$ & $1.5 \pm 16$ & $2.8 \pm 17$ & $-2.1 \pm 12$ \\
Science identity (/100) & $5.3 \pm 14$ & $4.9 \pm 15$ & $6.3 \pm 10$ & $3.3 \pm 15$ & $4.3 \pm 15$ & $0.43 \pm 14$ \\
FMCE (/100) & $13 \pm 21$ & $11 \pm 17$ & $19 \pm 28$ & $12 \pm 14$ & $13 \pm 14$ & $11 \pm 14$ \\
\hline
\end{tabular}

the FMCE performance even better than the cohort of Study 1 , there were no significant effects of the experimental treatment across the range of outcomes. Despite our attempt to increase the personal relevance and "discussion" of underrepresentation of women in physics, neither women nor men showed any differential change in their identities as a result. It is perhaps noteworthy that across both studies on all outcomes, the gains in the treatment group were greater than those in the control group; however, in every case these are not statistically significant. It may be possible that the effect size of this experiment is too small to be detected, but may show up with larger sample sizes. (Note that we repeated the analysis with all participants together to check this but the results were still null except for the same marginal effect that treatment males out-gained control males on the FMCE.)

\section{IMPLICATIONS AND FUTURE DIRECTIONS}

Putting aside the aforementioned possibility that this type of exposure results in very small improvements in identity or performance (which might be detected by a much larger study), it appears that the necessary level of engagement in this type of discourse is greater than we have so far developed in these studies. Recall that we have seen in other work that women who report having been exposed to underrepresentation discussion do indicate that they are more interested in a physical science career [5, 1]. In other classroom observational studies [6] we have seen rich and meaningful examples of such discourse and its impacts on women. Thus, in future work, we will "build up" significant activities/lessons which more effectively approach the examples we have identified elsewhere. It remains to be seen what the "minimal impactful unit" of such discourse looks like.

\section{ACKNOWLEDGEMENTS}

Thanks to the study participants and Carrie Beattie, Mark Harmon, and Kylie Paige for assistance in earlier development of the identity instrument used in this paper. Also thanks to Dean Zollman for useful comments on partial results of these studies.

\section{REFERENCES}

[1] Z. Hazari, G. Sonnert, P. Sadler, and M. Shanahan, Journal of Research in Science Teaching 47, 978-1003 (2010).

[2] G. Potvin, and Z. Hazari, Proceedings of Physics Education Research Conference (2013).

[3] J. Cribbs, Z. Hazari, P. Sadler, and G. Sonnert, Psychology of Mathematics Education - North American (PME-NA) Chapter Conference (2012).

[4] A. Godwin, G. Potvin, Z. Hazari, and R. Locke, Proceedings of 2013 American Society for Engineering Education Annual Conference and Exposition (2013).

[5] Z. Hazari, G. Potvin, R. M. Lock, F. Lung, G. Sonnert, and P. M. Sadler, Physical Review Special Topics-Physics Education Research 9, 020115 (2013).

[6] R. Lock, R. Tompkins, and Z. Hazari, APS April Meeting (2013).

[7] R. K. Thornton, and D. R. Sokoloff, American Journal of Physics 66, 338-352 (1998).

[8] C. Steele, American Psychologist 52, 613-629 (1997).

[9] I. Dar-Nimrod, and S. J. Heine, Science 314, 435-435 (2006).

[10] G. Potvin, C. Beattie, and K. Paige, American Association of Physics Teachers Summer Conference (2011).

[11] R Core Team, $R:$ A Language and Environment for Statistical Computing, R Foundation for Statistical Computing (2014), URL http: //www.R-project.org/. 